\title{
Morphological features and mechanics of destruction of materials with different structures under impact drop cyclic loading
}

\author{
Valery N. Varavka ${ }^{1,}$, Oleg V. Kudryakov ${ }^{1}$, Igor Yu. Zabiyaka ${ }^{1}$, and Antonina A. Shapovalova ${ }^{1}$ \\ ${ }^{1}$ Don State Technical University, Material Science Department, 344000 Gagarin sq. 1, Rostov-on-Don, Russia
}

\begin{abstract}
The process of erosion destruction of steels with austenitic, sorbitol and martensitic structure under the influence of high-speed liquid-drop collisions is studied. The characteristics of the morphological features and mechanisms of the process of surface degradation of steels with different structures are given. Their classification criterion is proposed on the basis of the diagrams of limiting states. Based on the theory of Paris-Erdogan, the computational and analytical model of the fatigue fracture of martensitic steel has been developed.
\end{abstract}

\section{Introduction}

The impact of a water drop on a metal surface includes several components: mechanical, cavitational (hydrodynamic and bubble) and corrosive [1-5]. The prolonged cyclic action of droplets causes fatigue failure of the metal, which takes place in several stages $[6,7]$. The first of these, incubation, is characterized by the fact that there are no signs of destruction and loss of mass at this stage. Therefore, the duration of the incubation period is usually taken as an anti-erosion property of the material under the conditions of droplet impact. In practice, the conditions for droplet erosion are formed during condensation in vapor-gas environments and lead to progressive deterioration of the blades of steam-gas turbine and compressor equipment. This problem is very relevant and is being actively studied [8-14].

To date, the physical nature of the initiation of wear during impact-dropping effects is not fully understood. In a number of works [3, 15-19] we have studied the features of this phenomenon in such ductile metallic materials as single-phase titanium alloys, steel with an austenitic or sorbitol structure.

The beginning of wear in this case is determined by the development of subsurface fatigue porosity (Fig. 1a), which appears in the metal in the impact zone by the percolation mechanism [20, 21], followed by pore fusion and the formation of primary surface erosional wear craters (Fig. 1b). In this case, the craters have a distinctive morphology with a very branched wall surface. Morphological features of such wear and its calculation and analytical model, developed on the basis of the principles of the fatigue theory of Goodman, are most thoroughly represented in the work [3]. In hardened metal materials, the percolation mechanism

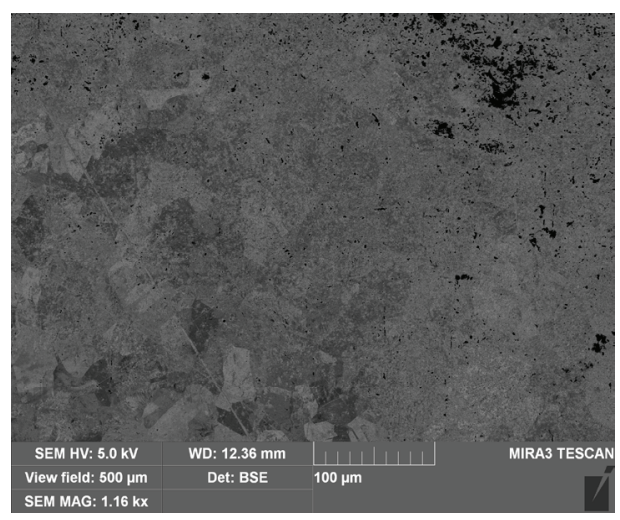

a)

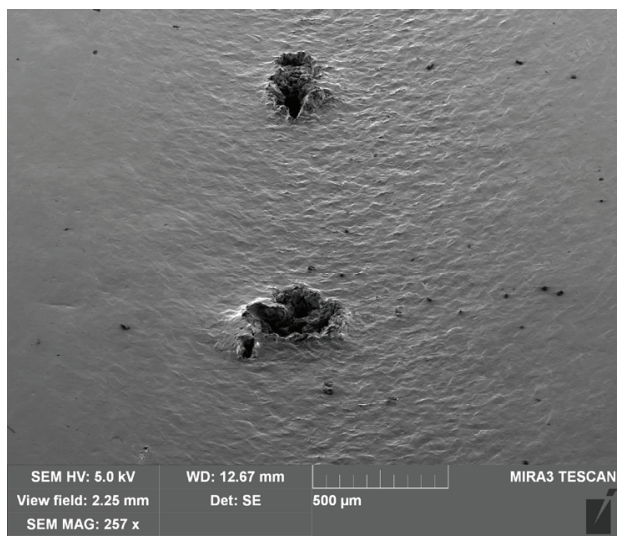

b)

Fig. 1. Development of wear during the droplet erosion of samples of austenitic steel $08 \mathrm{Cr} 18 \mathrm{Ni} 10 \mathrm{Ti}$ (SEM): a - formation of fatigue porosity at the collision site (cross section of the sample in depth); $b$ - formation of the first erosion craters (erosion track on the sample surface)

\footnotetext{
* Corresponding author: varavkavn@g,gmail.com
} 
cannot be realized, since the size of the plasticity zones is too small. In this case, the fatigue porosity is not formed, and the morphology of the primary wear craters is determined by the mechanism of nucleation, growth, and branching of brittle fracture fatigue cracks. Distinctive features of such a mechanism for the nucleation of erosion craters are the relatively smooth surface of their walls (Fig. 2a) and the presence of cracks in the cross section of the craters (Fig. 2b). On the basis of these experimental data, the main task of the present work was to find regularities and create a computational and analytical model of erosion wear of such materials. At the same time, the problem of criterial evaluation of materials was solved, with the help of which they could be categorized as plastic or hardened in order to be described within the corresponding mechanical model of fatigue failure.

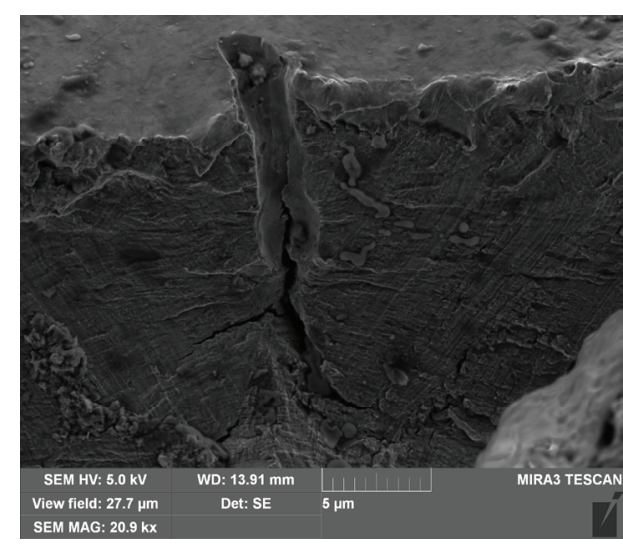

a)

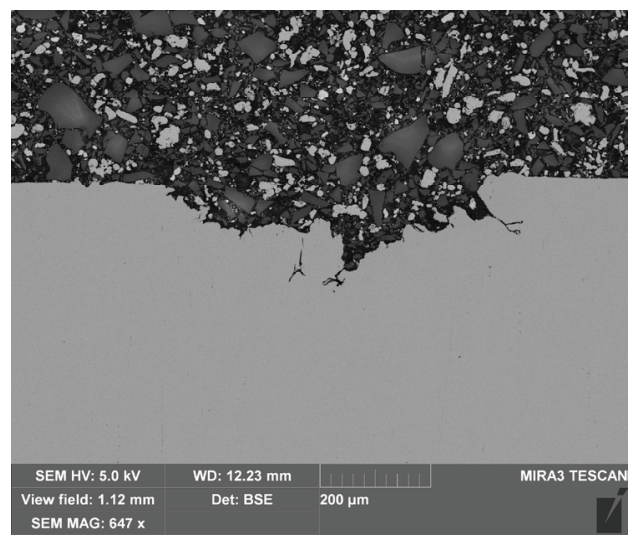

b)

Fig. 2. Erosion craters on the surface of a sample of martensitic steel $20 \mathrm{Cr} 13$ (SEM): a - the wall of the primary crater with a branched sharp crack; $b$ - cross section of the crater with fatigue cracks

\section{Limiting state diagrams}

Analysis of experimental data obtained from the results of bench erosion testing of steel samples with different structures makes it possible to recommend their limiting state diagrams as a classification criterion for ductile and durable materials. Such diagrams of austenitic and martensitic steels are presented in Fig. 3 and Fig. 4, respectively. The linear graph represents the limiting, and the nonlinear - the critical stress. Austenitic steel does not have points of intersection on the diagram (Fig. 3 ), which indicates the absence of cracks and allows it to be categorized as ductile materials with percolation fatigue failure mechanisms under conditions of droplet impingement erosion wear. The points of intersection of the graphs in the martensitic steel diagram (Fig. 4) are the initial $l_{0}$ and critical $l_{C}$ fracture dimensions by their physical entity. For $l<l_{0}$, the crack does not spontaneously grow, and there is no danger of brittle failure.

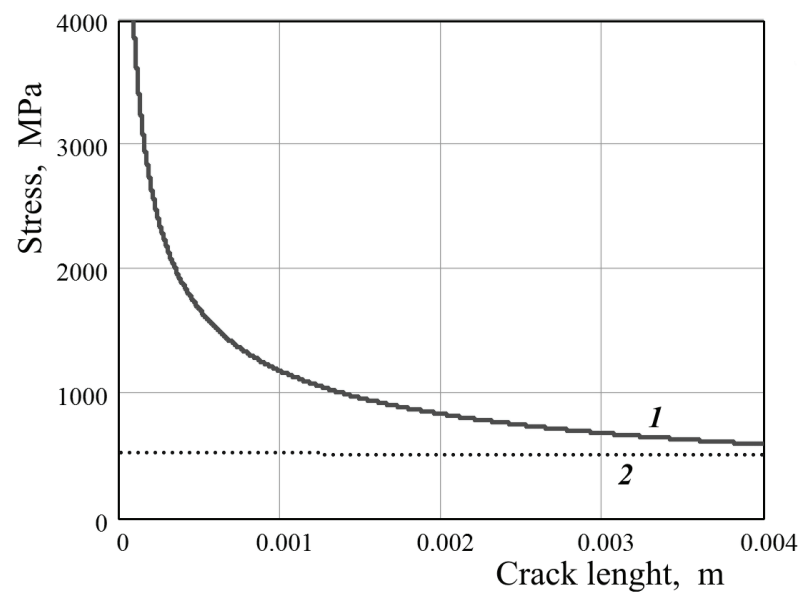

Fig. 3. The diagram of the limiting states of austenitic steel 08Cr18Ni10Ti.

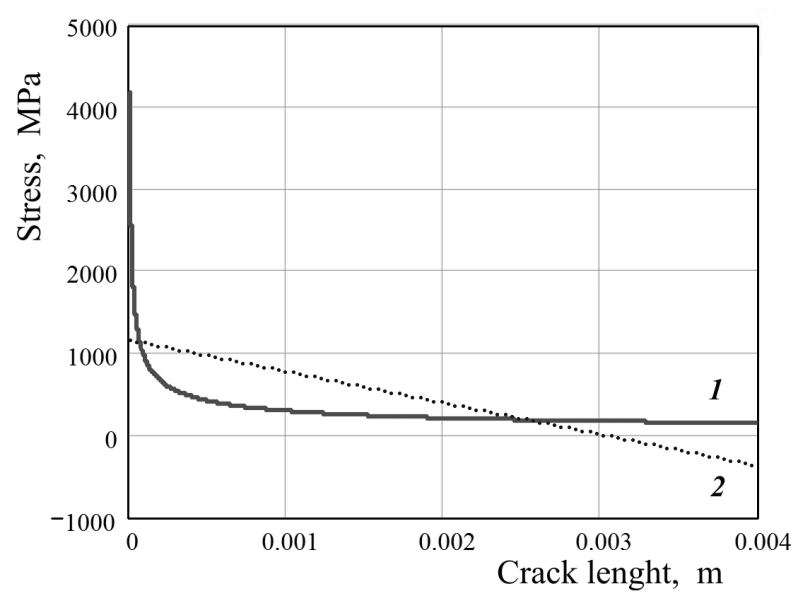

Fig. 4. The diagram of the limiting states of steel $20 \mathrm{Cr} 13$ with a martensitic structure

At $l \geq l_{C}$, crack growth is irreversible and autocatalytic is accelerated, and destruction (formation of an erosion wear crater) is developing catastrophically fast. The values of $l_{0}$ and $l_{C}$ in the framework of the proposed calculation and analytical model are found by solving equations (1) for ultimate $\sigma_{p}$ and critical $\sigma_{k}$ stresses for a material specified by such properties as hardness $H V$, tensile strength $\sigma_{B}$, fracture toughness $K_{I C}$ taking into account the geometric parameters of the crack $Y$. The factor $Y$ characterizes the type of crack and can be determined from directories, for example [22]. In this case, depending on the choice of the crack type, the 
values of $Y$ can differ substantially and they must be coordinated with the experiment. For example, for steel $20 \mathrm{Cr} 13$ with a martensitic structure, we have: $l_{C}=68 \mu \mathrm{m}$ - for the projection of the crack on the surface of the sample and $l_{C}=7 \mu \mathrm{m}-$ for the projection of the crack along the sample section (depth).

$$
\sigma_{p}(l)=H V-\frac{\sigma_{B}^{3}}{4 \cdot K_{1 C}^{2}} \cdot l ; \quad \sigma_{k}(l)=\frac{K_{1 C}}{Y \cdot \sqrt{\pi \cdot l}}
$$

In the interval between $l_{0}$ and $l_{C}$, the rate of crack growth (per one loading cycle, i.e, one dropping impact) is determined in accordance with Paris equation (2) of the Paris-Erdogan fatigue theory [23]. To use the methodical apparatus of this theory, it is necessary to experimentally determine the parameters of the Paris equation $m$ and $A$, which are the empirical constants of the material. Thus, for example, bench tests performed by us on samples of steel $20 \mathrm{Cr} 13$ with a martensitic structure for the conditions of droplet impingement erosion give the values of the parameters of the Paris equation: $m=2.5$ and $A=3.403 \cdot 10^{-9} \mathrm{~m} /$ cycle (m/ impact).

$$
\frac{d l}{d N}=A \cdot \Delta K^{m}
$$

where the value of $\Delta K$ is determined by the expression:

$$
\Delta K=K_{\max }-K_{\min }=\Delta \sigma \cdot \sqrt{\pi \cdot l}
$$

taking into account the following conditions:

$K_{\max }=\sigma_{\max } \sqrt{\pi \cdot l}$ and $K_{\min }=\sigma_{\min } \sqrt{\pi \cdot l}$ for $\sigma_{\min }>0$. Here $K$ is the stress concentration coefficient for the corresponding maximum $\sigma_{\max }$ and minimum $\sigma_{\min }$. stresses of a single cycle.

\section{Single loading cycle}

It was shown in [3] that when a liquid drop hits a metal surface, the stress $\sigma$ is distributed over the surface in time $\tau$ in accordance with the graph in Fig. 5. The value $\sigma$ is expressed in relative units $\rho_{0}{ }^{\bullet} c_{0} \bullet V_{0}$, called the hydraulic shock margin. Time $\tau$ also has a relative scale, where $\rho_{0}-$ is the density of the liquid, $c_{0}-$ is the velocity of sound in the liquid under normal conditions, $V_{0}$ is the impact velocity, and $R_{0}$ is the radius of the drop. Fig. 5 also shows the stress values adopted in the theory of fatigue, in particular in the Paris-Erdogan theory (maximal $\sigma_{\max }$, minimum $\sigma_{\min }$, mean $\sigma_{m}$, etc.). However, they need to be refined for use in the Paris equation (2).

\section{Multi-cycle loading}

Fig. 5 shows the distribution of stresses for a single impact, and the Paris equation is used for multi-cycle loading. In a single impact with an increase in the time $\sigma_{\text {min }} \rightarrow 0$, the single loading cycle becomes repeatingstress cycle. And the time between impacts falling into one point exceeds several times the time of a single impact.

With a repeating-stress cycle in the formula (3) in accordance with Fig. 5 it should be taken that

$$
\Delta \sigma=\sigma_{\max }=0,528 \cdot \rho_{0} \cdot c_{0} \cdot V_{0} .
$$

By the solution of the Paris equation (2) for the conditions of droplet impingement erosion, an acceptable number of cycles (droplet impacts) $N_{C}$ that determines the duration of the incubation period of erosive wear of this material can be found:

$$
N_{C}=\int_{0}^{N_{C}} d N=\int_{l_{0}}^{l_{C}} \frac{d a}{A \cdot(\Delta K)^{m}}
$$

Strictly speaking, the value of $N_{C}$ obtained by integration is not the value of the incubation period of droplet impingement erosion $m_{0}$, since it does not take into account the number of $N_{3}$ cycles necessary to form a crack of size $l_{0}$. Therefore, for application of expression (5), correlations of the $N_{C}$ value with the bench test data are necessary, taking into account that $m_{0}=N_{3}+N_{C}$.

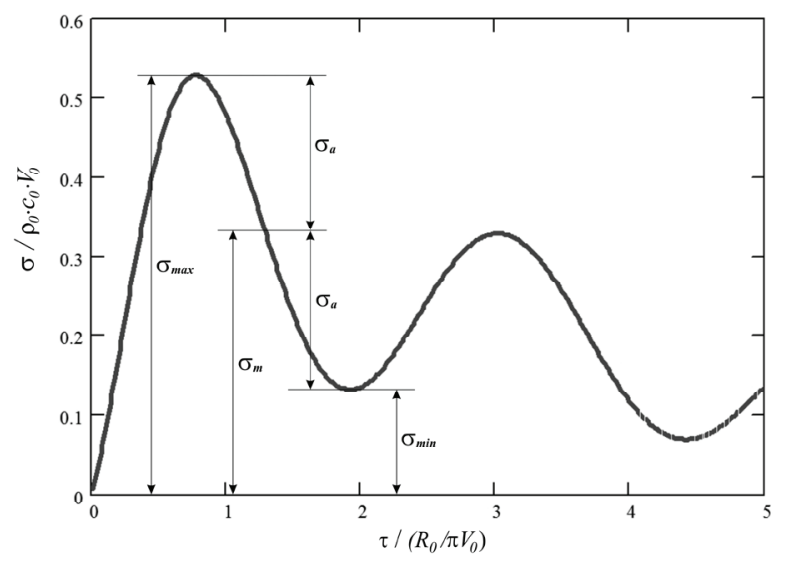

Fig. 5. Calculated parameters of the loading cycle for a drip collision

Without going into the details of this correlation, which are fulfilled in the development of the computational and analytical model, we present the result of the use of the Paris-Erdogan theory $(2)+(3)+(5)$, taking into account the features of the droplet impingement erosion mechanism (4) using the example of steel $20 \mathrm{Cr} 13$ with a martensitic structure, presented in Fig. 6. Dependences in Fig. 6 can be considered as the applied criterion of erosion failure, showing the minimum value of the impact velocity at which wear in steel is initiated. If the graph of the function in Fig. 6 is below the threshold line, the distance over which the crack propagates in one loading cycle lies in the region of elastic deformations and elastically relaxes after the load is removed. In this case, the crack will not grow. For example, it follows from Fig. 6 that if there are already cracks in the martensite of steel $20 \mathrm{Cr} 13$ of length $l_{0}=1.5 \mu \mathrm{m}$ (line 2), then they will develop at the impact velocities $V_{0}$ exceeding $150 \mathrm{~m} / \mathrm{s}$. 


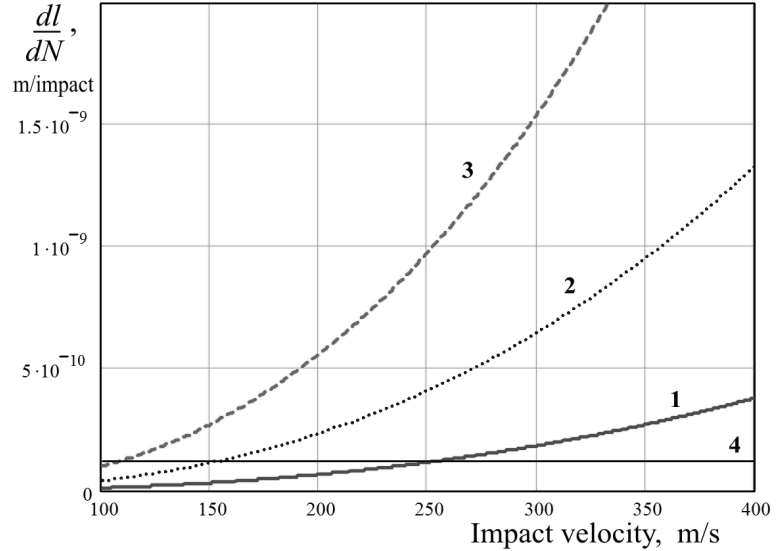

Fig. 6. The dependence of the rate of crack growth in steel $20 \mathrm{Cr} 13$ with a martensitic structure on the impact velocity for different values of the initial crack length: 1- $0.55 \mu \mathrm{m} ; 2-1.5 \mu \mathrm{m}$; $3-3.0 \mu \mathrm{m} ; 4$ - threshold value of growth of a sharp crack

\section{Conclusion}

Thus, the criterion of the minimum impact velocity at which wear is initiated can be considered as universal for any material. A similar criterion was proposed in [3] on the basis of the Goodman fatigue model for plastic metal alloys, and in this paper it was used for reinforced (hardened) metal alloys based on the Paris-Erdogan model. This provides a single measuring space for materials, the surface degradation of which during droplet impingement erosion occurs in fundamentally different mechanisms - by developing brittle cracks in hardened metal materials and by forming fatigue porosity by the mechanism of percolation in ductile metal materials.

The reported study was funded by RFBR in the framework of the research project no. 15-08-06181.

\section{References}

1. A. Chizhov, A. Shmidt, Tech. Phys., 45, 1529 (2000)

2. K. Haller, Y. Ventikos, D. Poulikakos, P. Monkewitz, J. Appl. Phys., 92, 2821 (2002)

3. V. Varavka, O. Kudryakov, J. Friction and Wear, 36 (1), 71 (2015)

4. V. Varavka, O. Kudryakov, J. Friction and Wear, 36 (2), 153 (2015)

5. O. Kudryakov, V. Varavka, V. Irkha, I. Moiseeva, Vestnik Don St. Tech. Univ., 17 (2), 56 (2017)

6. V. Varavka, O. Kudryakov, Al. Mednikov, V. Irkha, Izv. Vyssh. Uchebn. Zaved. Sev. Kavkaz. Reg. Tekhn. Nauki, 6, 92-98 (2011)

7. V. Ryzhenkov, G. Kachalin, A. Mednikov, Al. Mednikov, O. Kudryakov, V. Varavka, Nadezhn. Bezopasn. Energ., 16 (1), 67 (2012)

8. S. Hattori, M. Takinami, Wear, 269, 310 (2010)

9. V. Ryzhenkov, A. Lebedeva, Al. Mednikov, Therm. Eng., 58 (9), 713 (2011)
10. A. Mednikov, V. Ryzhenkov, L. Seleznev, A. Lebedeva, Therm. Eng. 59 (5), 414 (2012)

11. E. Tobin, T. Young, D. Raps, O. Rohr, Wear, 271, 2625 (2011)

12. S. Sandeep, Int. J. Mech. Eng. \& Rob. Res., 1 (3), 214 (2012)

13. N. Kamkar, F. Bridier, P. Bocher, P. Jedrzejowski, Wear of Materials, 301 (1-2), 442 (2013)

14. A.S. Vasiliev, S.S. Volkov, A.A. Belov, S.Yu. Litvinchuk, S.M. Aizikovich, Int. J. Eng. Sci., 112, 7 (2017)

15. V. Varavka, O. Kudryakov, Vestnik Don St. Tech. Univ., 11 (8), 1376 (2011)

16. O. Kudryakov, V. Varavka, Materialoved. (5), 36-43 (2012)

17. O. Kudryakov, V. Varavka, Materialoved. (6), 14-19 (2012)

18. V. Varavka, O. Kudryakov, Izv. Vyssh. Uchebn. Zaved. Sev.Kavkaz. Reg. Tekhn. Nauki, (3), 45-50 (2012)

19. O. Kudryakov, V. Varavka, Uprochn. Tekhnol., Pokr., (10), 40-47 (2012)

20. M. Shtremel', Durability of Alloys. Ch. 2. Deformation, Moscow: Mos. Inst. Stali \& Splavov, 527 (1997)

21. M. Ashby, D. Jones, Engineering Materials, Pergamon, 142 (1989)

22. Y. Ito, Y. Murakami, N. Hasebe, R. Yuki, M. Toya and others, Handbook on stress intensity factors, Pergamon, 90-107 (1987; Mir, 1990)

23. P. Paris, F. Erdogan, Trans. ASME, J. Basic Engng., 15, $528(1963)$ 